\title{
A new ambipolar blue emitter for NTSC standard blue organic light-emitting device
}

\author{
Tsung-Cheng Tsai ${ }^{a}$, Wen-Yi Hung ${ }^{a, *}$, Liang-Chen Chi ${ }^{b}$, Ken-Tsung Wong ${ }^{b, *}$, \\ Cheng-Chih Hsieh ${ }^{\mathrm{b}}$, Pi-Tai Chou ${ }^{\mathrm{b}, *}$
}

a Institute of Optoelectronic Sciences, National Taiwan Ocean University, Keelung 202, Taiwan

${ }^{\mathrm{b}}$ Department of Chemistry, National Taiwan University, Taipei 106, Taiwan

\section{A R T I C L E I N F O}

\section{Article history:}

Received 22 August 2008

Received in revised form 21 October 2008

Accepted 21 October 2008

Available online 5 November 2008

\section{PACS:}

73.50.-h

73.61.Ph

78.55.Qr

78.60.Fi

78.66.Qn

Keywords:

Organic light-emitting diodes

Deep blue emitter

Ambipolar transport

Coplanar molecule

\begin{abstract}
A B S T R A C T
A novel blue emitter, In2Bt, featured with a rigid and coplanar distyryl-p-phenylene backbone flattened by two different bridging atoms (i.e. carbon and sulfur) exhibits high thermal and morphological stability $\left(T_{\mathrm{g}} \sim 192{ }^{\circ} \mathrm{C}\right)$ and ambipolar charge carrier mobilities in the range of $10^{-4} \sim 10^{-5} \mathrm{~cm}^{2} \mathrm{~V}^{-1} \mathrm{~s}^{-1}$. OLED device: ITO/PEDOT:PSS (300 $\AA$ )/ $\alpha$-NPD $(200 \AA) /$ TCTA $(100 \AA) /$ In2Bt $(200 \AA) /$ TPBI $(500 \AA) / L i F(5 \AA) / A l(1500 \AA)$ utilized In2Bt as an emitter gave a maximum brightness as high as $8000 \mathrm{~cd} \mathrm{~m}^{-2}(12 \mathrm{~V})$ and saturated-blue emission with CIE chromaticity coordinates of $(0.16,0.08)$, which is very close to the National Television Standards Committee (NTSC) standard blue gives an enlarged palette of colors for color displays.
\end{abstract}

(c) 2008 Elsevier B.V. All rights reserved.

\section{Introduction}

In recent years, the development of deep blue emitters for OLED, which can match the National Television System Committee (NTSC) standard blue CIE $(x, y)$ coordinates of $(0.14,0.08)$ has drawn considerable attentions [1-5]. Such a device not only can effectively reduce the power consumption of a full-color OLED but also can be utilized to generate emission of other colors through energy transfer. However, the molecular design for a deep blue emitter is

\footnotetext{
* Corresponding authors. Tel.: +8862 33661665; fax: +886233661667 (K.-T. Wong).

E-mail addresses: wenhung@mail.ntou.edu.tw (W.-Y.Hung), kenwong@ ntu.edu.tw (K.-T. Wong).
}

quite challenging in terms of molecular structures. Molecules that can emit deep blue light normally have a restricted $\pi$-conjugated length, which consequently limits the spaces for tailoring the desired chromophore with sufficient thermal and thin film morphological stability. Thus, only few materials can meet these requirements so far [15]. Recently, the ladder-type oligomers and polymers [614], due to their rigid and coplanar structures which can enhance the $\pi$-conjugation, charge mobility, and luminescence intensity have emerged as potential materials for optoelectronic applications. In this regard, the physical properties and possible applications of ladder-type oligoor $\operatorname{poly}(p$-phenylene)s with various heteroatom bridges have been extensively studied [15-18]. More importantly, the physical properties of ladder-type materials can be 
tailored by modulating the degree of conformational flexibility between consecutive backbone units or, more effectively, manipulating the electronic properties by flattening the $\pi$-conjugated molecular framework with heteroatoms. In this paper, we report a new blue lightemitting material based on a coplanar molecular structure, in which benzothiophene was fused to neighboring phenylene ring through intramolecular annulation via $\mathrm{sp}^{3}$ hybridized carbon atoms bearing two p-tolyl groups as peripheral substituents [19]. The non-doped OLED device shows an external quantum efficiency of $1.3 \%$ and saturated-blue emission with CIE chromaticity coordinates $(0.16,0.08)$, which is almost the National Television Standards Committee (NTSC) standard blue.

\section{Results and discussion}

Fig. 1 depicts the molecular structure of blue emitter: 7,14-dihydro-7,7,14,14-tetrakis(4-methylphenyl)-s-indaceno[1,2-b:5,6-b']bis[1]benzothiophene (In2Bt) featured with a rigid and coplanar distyryl-p-phenylene backbone flattened by two different bridging atoms (i.e. carbon and sulfur). The synthesis and X-ray structural analysis of In2Bt have been reported by our group previously [19]. The peripheral $p$-tolyl substituents occupy the top and bottom faces of the main conjugated backbone prevent undesirable aggregation and improve thermal and morphological stability efficiently. We characterized the morphological and thermal properties of In2Bt using differential scanning calorimetry (DSC) and thermogravimetric analyses (TGA), respectively. In2Bt exhibits a distinct glass transition temperature $\left(T_{\mathrm{g}} \sim 192^{\circ} \mathrm{C}\right)$, which allows to form homogeneous and stable amorphous films upon thermal evaporation, a critical issue for OLED application. We ascribed the amorphous behavior and high values of $T_{\mathrm{g}}$ to the rigidity of the conjugated backbone and the presence of the $p$-tolyl substituents, which can effectively suppress intermolecular interactions and crystallization. The aryl substituents impart the oligomers with a high tolerance to heat, as indicated by their high decomposition temperatures $\left(T_{\mathrm{d}} \sim 408^{\circ} \mathrm{C}\right.$, corresponding to a $5 \%$ weight loss).

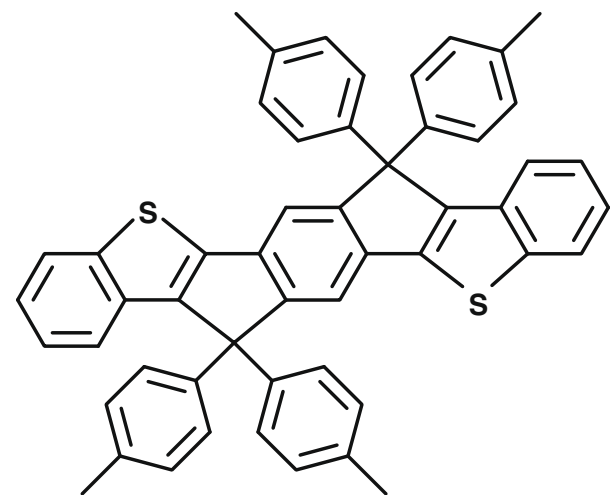

Fig. 1. Chemical structure of In2Bt.

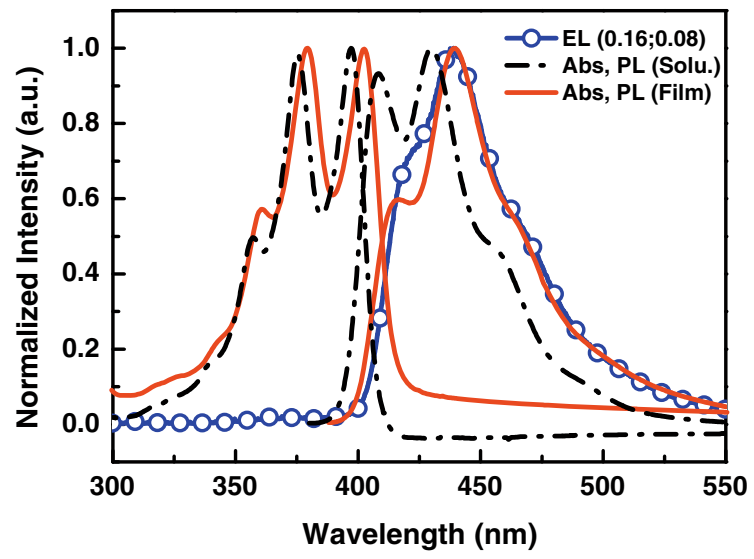

Fig. 2. The optical absorption, photoluminescence (PL) spectra of In2Bt in solution and thin film, and the electroluminescence (EL) spectra of device using In2Bt as an emitter.

Fig. 2 summarizes the electronic absorption (Abs) and photoluminescence (PL) spectra of In2Bt in chloroform solution and as solid films. Due to the coplanar conformation of the main chromophore, the absorption spectra of In2Bt in dilute solution and in thin film are almost superimposable with slightly red-shifted maxima in solid state (Abs $\lambda_{\max }$ in solution, $375,397 \mathrm{~nm}$, and in film, 385 , $402 \mathrm{~nm}$ ). The thin film emission spectra of In2Bt exhibit slightly red-shifted maxima (PL $\lambda_{\max }$ in solution 408 , $430 \mathrm{~nm}$, and in film 416, $439 \mathrm{~nm}$ ). In addition, the small Stokes shifts ( $\Delta \lambda \sim 11 \mathrm{~nm}$ in solution) and the mirror images of the absorption and emission spectra are consistent with the molecular rigidity observed in the solid state structures $[19,20]$. We estimated the $\pi-\pi^{*}$ band gap $\left(\Delta E_{\mathrm{g}} \sim 3 \mathrm{~V}\right)$ from the lowest energy absorption edges (ca. $413 \mathrm{~nm}$ ) in the UV-Vis absorption spectra. The thin film photoluminescence quantum yield (PLQY) of In2Bt measured by a calibrated integral sphere (HAMAMATSU C9920) is ca. $14 \%$. In order to gain more insight into the low PLQY of In2Bt in thin film, we have employed timecorrelated single photon counting measurement with a time resolution of $\sim 30 \mathrm{ps}$. Upon excitation at $390 \mathrm{~nm}$ and monitoring the emission at e.g. $450 \mathrm{~nm}$, the observed lifetime $\left(\tau_{\text {obs }}\right)$ for In2Bt in thin film was determined to be 268 ps. From the PLQY and observed lifetime, the radiative $\left(k_{\mathrm{r}}\right)$ and non-radiative $\left(k_{\mathrm{nr}}\right)$ decay rate constants, deduced by Eq. (1), were $5.2 \times 10^{8} \mathrm{~s}^{-1}$ and $3.2 \times 10^{9} \mathrm{~s}^{-1}$, respectively,

$\operatorname{PLQY}(\Phi)=\frac{\tau_{\mathrm{obs}}}{\tau_{\mathrm{r}}}=\frac{k_{\mathrm{r}}}{k_{\mathrm{obs}}}=\frac{k_{\mathrm{r}}}{k_{\mathrm{r}}+k_{\mathrm{nr}}}$.

Normally, a skeleton possessing a rigid distyryl-p-phenylene backbone is expected to provide a reasonably high PLQY. The large $k_{\mathrm{nr}}$ (c.f. $k_{\mathrm{r}}$ ) value and hence low PLQY for In2Bt in thin film is of fundamental interest and, to our viewpoint, can be tentatively attributed to two factors associated with the sulfur atom and/or the molecular framework. On one hand, sulfur atoms embedded inside the chromophore backbone may facilitate the non-radiative processes due to the heavy atom effect, a common phenomenon occasionally reported in sulfur-containing oligoaryls [21]. On the other hand, the soft sulfur atom pos- 


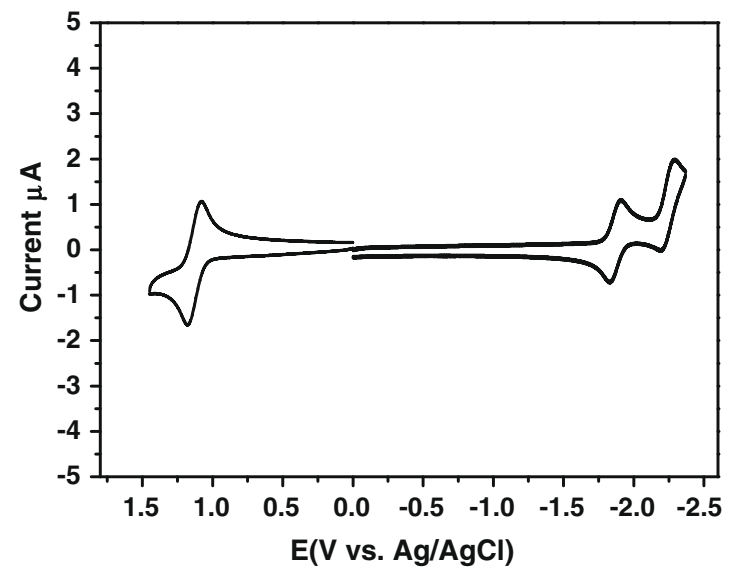

Fig. 3. The cyclic voltammogram of In2Bt.

sesses a lower lying non-bonding orbital that may facilitate the non-radiative process via certain vibronic coupling modes. Such modes may be associated with the slightly twisted molecular plane of In2Bt in thin film [12]. At room temperature or upon cooling to $77 \mathrm{~K}$, phosphorescence of the In2Bt thin film was obscure. Therefore, the former case of heavy atom enhanced spin-orbit coupling and hence the intersystem crossing is less likely. Nevertheless, at this stage, the actual deactivation mechanism is still pending for resolution. Note we also cannot eliminate the possibility that relatively low PLQY, in part, is attributed to the reabsorption problem occurring in solid states.

Fig. 3 depicts the electrochemical properties of In2Bt studied by cyclic voltammetry $(\mathrm{CV})$. We detected one reversible oxidation potential occurred at $1.13 \mathrm{~V}$ (V vs. $\mathrm{Ag} / \mathrm{AgCl}$, in $\mathrm{CH}_{2} \mathrm{Cl}_{2}$ using a glassy carbon electrode with $0.1 \mathrm{M}$ of $n \mathrm{Bu}_{4} \mathrm{NPF}_{6}$ as electrolyte) and one reversible reduction potential at $-1.87 \mathrm{~V}$ together with one quasireversible reduction at $-2.25 \mathrm{~V}$ ( V vs. $\mathrm{Ag} / \mathrm{AgCl}$ in dimethylforamide using a glassy carbon electrode with $0.1 \mathrm{M}$ of $n \mathrm{Bu}_{4} \mathrm{ClO}_{4}$ as electrolyte). Thus, In2Bt exhibits bipolar character with excellent electrochemical stability. The HOMO energy level of In2Bt (ca. $-5.7 \mathrm{eV}$ ) was calculated using a linear correlation with $\alpha-\mathrm{NPB}(\mathrm{HOMO}=\mathrm{ca} .-5.3 \mathrm{eV})$, which exhibits an $E_{1 / 2}^{\mathrm{OX}}$ at $0.74 \mathrm{~V}$ measured under the same conditions [22]. And the corresponding LUMO (ca. $-2.7 \mathrm{eV}$ ) of In2Bt was then derived by subtracting the corresponding optical band gap from the HOMO energy.

We used time-of-flight (TOF) techniques with a device structure of glass/Ag $(30 \mathrm{~nm}) / \mathbf{I n 2 B t}(2.1 \mu \mathrm{m}) / \mathrm{Ag}(200 \mathrm{~nm})$ to measure the charge carrier mobilities of In2Bt [23]. Fig. $4 \mathrm{a}$ and $\mathrm{b}$ shows representative TOF transients for electrons and holes of under an applied field, respectively. The TOF transients reveal that In2Bt exhibits slightly dispersive carrier-transport characteristics. In the double-logarithmic representation (inset of Fig. $4 a$ and b), the carrier-transit time, $t_{\mathrm{T}}$, needed for determining carrier mobilities can be clearly extracted from the intersection point of two asymptotes. The field dependence of hole mobility $\mu$ thus determined (Fig. 4c) from the transit time $t_{\mathrm{T}}$ according to the relation $\mu=d^{2} / V t_{\mathrm{T}}$, where $d$ is the sample thickness and $V$ is the applied voltage. Apparently,
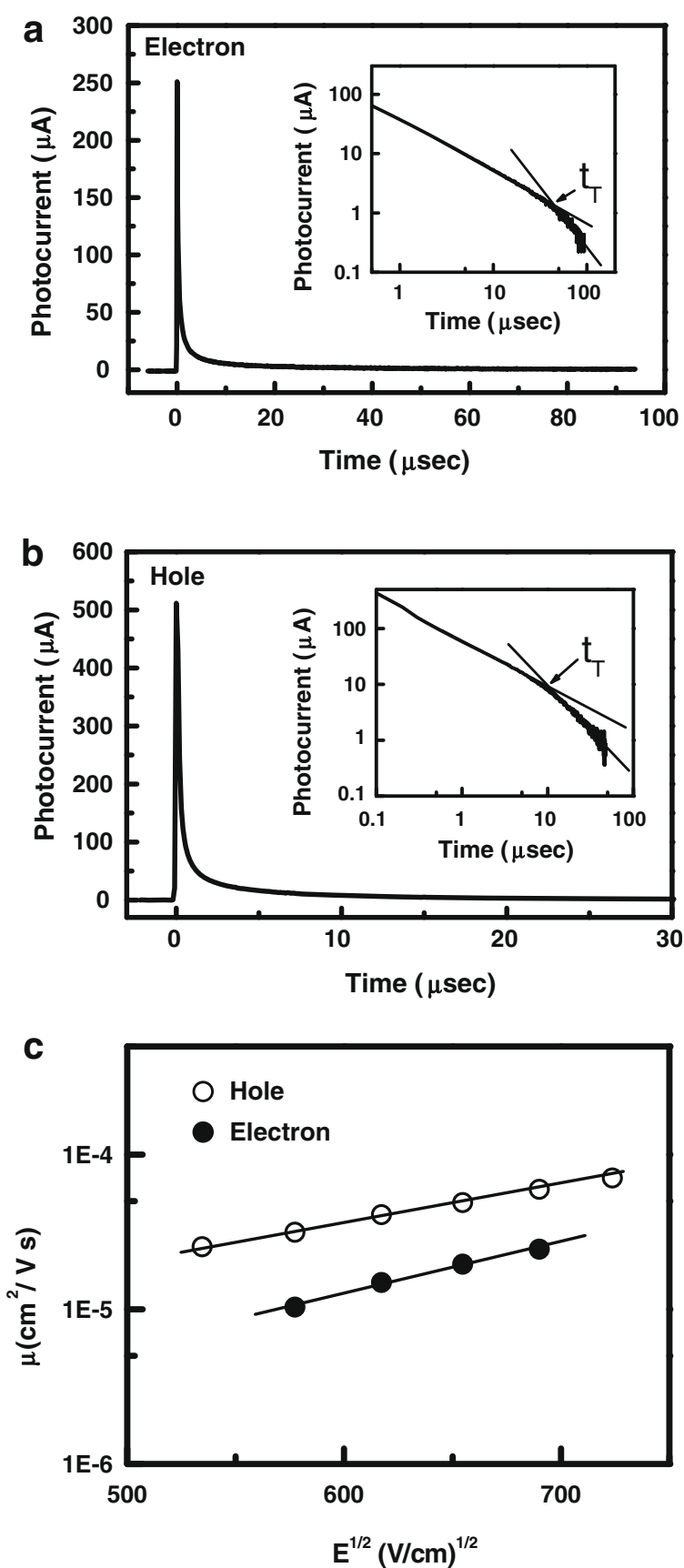

Fig. 4. TOF transients for In2Bt $(2.1 \mu \mathrm{m}$ thick): (a) electron, $E=4.8 \times 10^{5} \mathrm{~V} \mathrm{~cm}^{-1}$; (b) hole, $E=4.3 \times 10^{5} \mathrm{~V} \mathrm{~cm}^{-1}$. Insets are the doublelogarithmic plots of (a) and (b). (c) Electron and hole mobilities vs. $E^{1 / 2}$.

In2Bt exhibits bipolar charge-transport properties, which has similar electron and hole mobilities in the range of $10^{-4} \sim 10^{-5} \mathrm{~cm}^{2} \mathrm{~V}^{-1} \mathrm{~s}^{-1}\left(E=3 \times 10^{5} \sim 5 \times 10^{5} \mathrm{~V} \mathrm{~cm}^{-1}\right)$ at room temperature.

To assess the feasibility of using In2Bt as an emitting material, multilayer device with the configuration of ITO/ PEDOT:PSS (300 ̊)/ $\alpha-N P D \quad(200 \AA) / T C T A \quad(100 \AA) / \mathbf{I n 2 B t}$ $(200 \AA) /$ TPBI $(500 \AA) / \operatorname{LiF}(5 \AA) / A l(1500 \AA)$ was fabricated. 


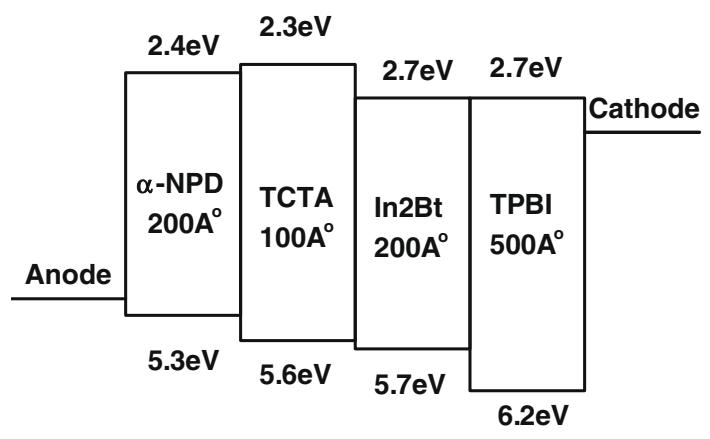

Fig. 5. HOMO and LUMO energy level diagram of the organic materials in the device.

Here, the conducting polymer, poly(ethylene dioxythiophene)/poly(styrene sulfonate) (PEDOT:PSS) is used as the hole-injection layer [24], 4,4'-bis( $N$-(1-naphthyl)- $N$ phenyl)biphenyldiamine ( $\alpha$-NPD) [25] and 4,4',4'-tri( $N$ carbazolyl) triphenylamine (TCTA) as hole-transport layers [26], In2Bt as an emitting layer, 1,3,5-tris( $N$-phenylbenzimidizol-2-yl)benzene (TPBI) [27] as an electron-transport and hole-blocking layer, LiF as an electron-injection layer and $\mathrm{Al}$ as a cathode, respectively. The energy levels alignment diagram of this blue OLED is shown in Fig. 5. The holes transport through the HTLs of $\alpha$-NPD and TCTA with a stepwise increase in HOMOs into the emitting layer, and
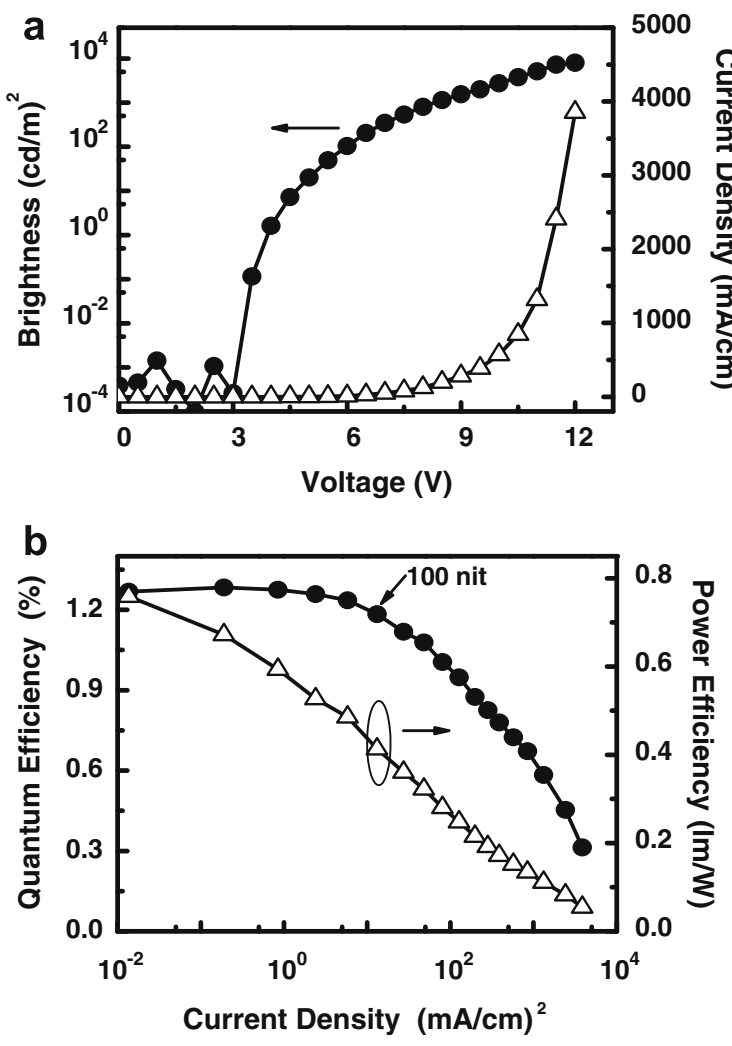

Fig. 6. (a) Brightness and current density vs. voltage. (b) External quantum efficiency and power efficiency vs. current density. subsequently are blocked by the higher energy barrier at the interface of In2Bt and TPBI. In the meantime, the electrons are injected into In2Bt layer from TPBI and blocked by the higher energy barrier at the interface of TCTA and In2Bt. Therefore, it is reasonable to anticipate that the recombination region is restricted only within the In2Bt layer. Consequently, a pure deep blue EL spectrum from In2Bt was obtained, which is corresponding well to the thin film PL spectrum (Fig. 2).

Fig. 6 a shows the current density and the luminance vs. voltage for the device. The device exhibits a rather low turn-on voltage of $3 \mathrm{~V}$ and low operation voltage $\left(100 \mathrm{~cd} \mathrm{~m}^{-2}\right.$ at $6 \mathrm{~V}, 1000 \mathrm{~cd} \mathrm{~m}^{-2}$ at $\left.8.5 \mathrm{~V}\right)$. The device exhibits unusual high currents $\sim 3860 \mathrm{~mA} \mathrm{~cm}^{-2}$ for $12 \mathrm{~V}$, giving a maximal brightness of $8000 \mathrm{~cd} \mathrm{~m}^{-2}$. Such high current density is ascribed to the well-matched energy levels of various functional layers used, rendering the device with a balanced holes and electrons recombination ratio. However, this device inevitably suffers from the re-absorption phenomena occurred in the solid states. The EL external quantum efficiency (EQE) shows a maximum at $1.3 \%$ $\left(0.86 \mathrm{~cd} \mathrm{~A}^{-1}\right)$ and a maximal power efficiency of $\sim 0.76 \mathrm{~lm} \mathrm{~W}^{-1}$ (Fig. 6b). It is worthy to note that the nondoped device shows a pure deep blue emission (Fig. 2) with CIE coordinates of $(0.16,0.08)$, which is almost perfectly match to the National Television Standards Committee (NTSC) standard blue value. The observed external quantum yield and luminance efficiencies is comparable to commonly non-doped deep blue devices, which warrants further investigations of analogous derivatives to afford materials with improved EL properties.

\section{Experimental}

\subsection{Device fabrication and measurement}

All chemicals were purified through vacuum sublimation prior to use. The OLEDs were fabricated by vacuum deposition of the materials at $10^{-6}$ torr. onto ITO-coated glass substrates having a sheet resistance of $15 \Omega$ /square. The ITO surface was cleaned through ultrasonication sequentially with acetone, methanol, and deionized water and then it was treated with UV-ozone. The deposition rate of each organic material was ca. 1-2 $\mathrm{As}^{-1}$. Subsequently, LiF was deposited at $0.1 \AA^{-1}$ and then capped with $\mathrm{Al}$ (ca. $5 \AA \mathrm{s}^{-1}$ ) by shadow masking without breaking the vacuum.

The current-voltage-brightness $(I-V-L)$ characteristics of the devices were measured simultaneously using a Keithley 6430 source meter and a Keithley 6487 picoammeter equipped with a calibration Si-photodiode. EL spectra were measured using an ocean optics spectrometer.

\subsection{Time-of-flight mobility measurements}

The samples for the TOF measurement were prepared by vacuum deposition using the structure: glass $/ \mathrm{Ag}$ $(30 \mathrm{~nm}) /$ organic $(2 \sim 3 \mu \mathrm{m}) / \mathrm{Al}(150 \mathrm{~nm})$, and then placed inside a cryostat and kept under vacuum. The thickness of organic films were monitored in situ with a quartz crys- 
tal sensor and calibrated by a profilometer (Tencor Alphastep 500). A pulsed nitrogen tunable dye laser was used as the excitation light source (to match the absorption of organic films) through the semitransparent electrode $(\mathrm{Ag})$ induced photogeneration of a thin sheet of excess carriers. Under an applied dc bias, the transient photocurrent was swept across the bulk of the organic film toward the collection electrode $(\mathrm{Al})$, and then recorded with a digital storage oscilloscope. Depending on the polarity of the applied bias, selected carriers (holes or electrons) are swept across the sample with a transit time of $t_{\mathrm{T}}$. With the applied bias $V$ and the sample thickness $D$, the applied electric field $E$ is $V / D$, and the carrier mobility is then given by $\mu=$ $D /\left(t_{\mathrm{T}} E\right)=\mathrm{D}^{2} /\left(V t_{\mathrm{T}}\right)$, in which the carrier-transit time, $t_{\mathrm{T}}$, can be extracted from the intersection point of two asymptotes to the plateau and the tail sections in double-logarithmic plots.

\section{Conclusions}

In summary, we reported a new deep blue emitter, In2Bt, which is based on a coplanar distyryl-p-phenylene core flattened through the incorporation of two different bridging atoms (i.e. carbon and sulfur). The promising physical properties of In2Bt including deep blue emission, high morphological stability, and bipolar charge-transport properties allow us to use it to achieve a non-doped deep blue device. The device fabricated use In2Bt as deep blue emitter leads to a NTSC pure blue electroluminescence ( $\mathrm{CIE}$ coordinates $=0.16,0.08$ ) with respectable device performances.

\section{Acknowledgement}

This study was supported financially by the National Science Council of Taiwan.

\section{References}

[1] Y.-H. Kim, D.-C. Shin, S.-H. Kim, C.-H. Ko, H.-S. Yu, Y.-S. Chae, S.-K. Kwon, Adv. Mater. 16 (2001) 1690.

[2] Y.-H. Kim, H.-C. Jeong, S.-H. Kim, K. Yang, S.-K. Kwon, Adv. Funct Mater. 15 (2005) 1799.

[3] Y.-H. Niu, B. Chen, T.-D. Kim, M.S. Liu, A.K.-Y. Jen, Appl. Phys. Lett. 85 (2004) 5433.

[4] Z.Q. Gao, Z.H. Li, P.F. Xia, M.S. Wong, K.W. Cheah, C.H. Chen, Adv. Funct. Mater. 17 (2007) 3194.

[5] S. Tang, M.R. Liu, P. Lu, H. Xia, M. Li, Z.Q. Xie, F.Z. Shen, C. Gu, H.P. Wang, B. Yang, Y. Ma, Adv. Funct. Mater. 17 (2007) 2869.

[6] S. Merlet, M. Birau, Z.Y. Wang, Org. Lett. 4 (2002) 2157.

[7] C. Xu, A. Wakamiya, S. Yamaguchi, J. Am. Chem. Soc. 127 (2005) 1638.

[8] J. Jacob, S. Sax, M. Gaal, E.J.W. List, A.C. Grimsdale, K. Müllen, Macromolecules 38 (2005) 9933.

[9] D. Vak, B. Lim, S.-H. Lee, D.-Y. Kim, Org. Lett. 7 (2005) 4229.

[10] A.C. Grimsdale, K. Müllen, Adv. Polym. Sci. 199 (2006) 1.

[11] D. Horhant, J.-J. Liang, M. Virboul, C. Poriel, G. Alcaraz, J. RaultBerthelot, Org. Lett. 8 (2006) 257.

[12] K.-T. Wong, L.-C. Chi, S.-C. Huang, Y.-L. Liao, Y.-H. Liu, Y. Wang, Org. Lett. 8 (2006) 5029

[13] F. Laquai, A.K. Mishra, M.R. Ribas, A. Petrozza, J. Jacob, L. Akcelrud, K. Müllen, R.H. Friend, G. Wegner, Adv. Funct. Mater. 17 (2007) 3231.

[14] Y. Wu, J. Zhang, Z. Bo, Org. Lett. 9 (2007) 4435.

[15] S.A. Patil, U. Scherf, A. Kadashchuk, Adv. Funct. Mater. 13 (2003) 609.

[16] S. Wakim, J. Bouchard, N. Blouin, A. Michaud, M. Leclerc, Org. Lett. 6 (2004) 3413.

[17] H. Ebata, E. Miyazaki, T. Yamamoto, K. Takimiya, Org. Lett. 9 (2007) 4499.

[18] L. Li, J. Xiang, C. Xu, Org. Lett. 9 (2007) 4877.

[19] K.-T. Wong, T.-C. Chao, L.-C. Chi, Y.-Y. Chu, A. Balaiah, S.-F. Chiu, Y.-H. Liu, Y. Wang, Org. Lett. 8 (2006) 5033.

[20] C. Poriel, J. Rault-Berthelot, F. Barriere, A.M.Z. Slawin, Org. Lett. 10 (2008) 373.

[21] K.R. Radke, K. Ogawa, S.C. Rasmussen, Org. Lett. 7 (2005) 5253.

[22] B.W. D’Andrade, S. Datta, S.R. Forrest, P. Djurovich, E. Polikarpov, M.E. Thompson, Org. Electron. 6 (2005) 11.

[23] P.M. Borsenberger, D.S. Weiss, Organic Photoreceptors for Imaging Systems, Marcel Dekker, New York, 1993.

[24] P. Strohriegl, J.V. Grazulevicius, Adv. Mater. 14 (2002) 1439.

[25] A. Berntsen, Y. Croonen, C. Liedenbaum, H. Schoo, R.J. Visser, J. Vleggaar, P. van de Weijer, Opt. Mater. 9 (1998) 125.

[26] Y. Kuwabara, H. Ogawa, H. Inada, N. Noma, Y. Shirota, Adv. Mater. 6 (1994) 677.

[27] J. Shi, C.W. Tang, C.H. Chen, U.S. Patent No. 5,646,948, 1997. 